\title{
Use of Laboratory Animals in A Pre-Clinical Trial: A Critical View of the Biomodels Development and Application
}

\author{
Gabriel Melo de Oliveira ${ }^{1 *}$, Cleiton Felizardo Brito ${ }^{1}$ and Ana Karina Furtado ${ }^{2}$ \\ ${ }^{1}$ Department of Cell Biology, Instituto Osvaldo Cruz, Brazil \\ ${ }^{2}$ Department of Animal Science, Instituto Osvaldo Cruz, Brazil
}

*Corresponding author: Gabriel Melo de Oliveira, Department of Cell Biology, Instituto Osvaldo Cruz, Rio de Janeiro, Brazil.

To Cite This Article: Gabriel Melo de Oliveira. Use of Laboratory Animals in A Pre-Clinical Trial: A Critical View of the Biomodels Development and Application. Am J Biomed Sci \& Res. 2019 - 4(2). AJBSR.MS.ID.000771. DOI: 10.34297/AJBSR.2019.04.000771

Received: July 12, 2019 | Published: July 23, 2019

\begin{abstract}
The scientific use of laboratory animals throughout history demonstrates changes in their vision and applicability, specifically from a moral, ethical and current legislation. In the Laboratory Animal Science Division of the Cellular Biology Laboratory, the development of experimental models and the performance of pre-clinical tests emphasizes the $3 \mathrm{Rs}$ principle of refinement in relation to equipment and methodologies. Thus, we transform the point of view over animal model as a Bioreactor for a Biomodel, being able to associate clinical/behavioral methods, consequently, to increase the welfare animal, reproducibility and confiability of the results.
\end{abstract}

Keywords: Experimental model; Animal welfare; Bioresearch

\section{Opinion}

The use of animal models in research has been done since Antiquity. In this period, Greek philosophers and thinkers like Hippocrates (450 BC) already related the aspect of sick human organs with that of animals, with clearly didactic purpose. The anatomists Alcmaeon (500 BC), Herophilus (330-250 BC) and Erasistratus (305-240 BC) performed animal vivisections with the purpose of observing structures and formulating hypotheses about the functioning associated with human organs. Subsequently, Galeno (129-210 AC) in Rome was perhaps the first to perform vivisection with experimental objectives, that is, to test variables through changes tested in animals. An importante (and curious) episode to establish limits to the use of animals in experimentation and teaching. This fact involved the wife and daughter of the French scientist Claude Bernard.

The great physiologist used, around 1860, his daughter's pet dog to teach his students. In response to this attitude, his wife founded the first association for the defense of laboratory animals. From this fact, Claude Bernard left many excellent texts on ethics for patients and also described that the scientist's posture could not be indifferent to the suffering and pain of the animals in the tests. The first publication on ethical aspects of the use of experimental animals was proposed in the United States by the American Medical Association in 1909. For many years research using animal models has not been strongly questioned because of its high social contribution, such as those enabled the development of anti-rabies vaccines, protection against tetanus and diphtheria. In 1959, zoologist William M. Russell and microbiologist Rex L. Burch published a book, where they established the Ethical Principle of the three "Rs" for animal research: Replace, Reduce, and Refine. This proposal does not avoid the use of animal models for experimentation, but it does make it humanized and appropriate form.

Laboratory Animal Science (LAS), current denomination for the use of animals for didactic and scientific purposes is not defined only by studies of the laboratory environment, but also in biomedical trials for the study of physiological, immunological, genetic, endocrinological and other animal bilogical mechanisms. Then we can say, LAS is based on the use of scientific, technological and toi innovation methodology, to investigate/implement materials, equipment, methodologies and management procedures that enable a better condition of the physiological, immunological, neuroendocrine and behavioral systems of laboratory animals. 
Critically analyzing this definition, we can assume that LAS has as its main basis the bioresearch and the maintenance of the animal welfare, as it would minimize possible discomfort and stress situations which, in turn, would affect the entire biological system, consequently would not influence the results of the pre-clinical trials. From this reasoning LAS would search to transform the idea that the laboratory animal is a Bioreactor for the Biomodel definition. The difference between the point of view of a laboratory animal as Bioreactor and Biomodel is subtle, but of great importance.

This difference is characterized by:

i. Bioreactor would be the laboratory animal used in scientific trials only for the purpose of evaluating a specific biological pathway, the course of infection of microorganisms or the efficacy of an experimental chemotherapy protocol. (especially when dealing with the use of genetically modified animals)

ii. Biomodel, we also use animal models for the respective approaches, such as genetic, physiological, and immunological studies, but the concept that the animal a complex biological system and a varied repertoire of physiological responses depend also on several factors that can directly influence the reproducibility and confiability of the results of the scientific and pre-clinical trials.
In this set of definitions, in conclusion, we can interpret that LAS has its main objective the search for all the ways to provide Biomodels kept in an animal facilities to minimize discomfort and stress of animals and consequently maintaining or enhancing the welfare of laboratory animals. However, from our point of view, we believe that only an intense work of sensitization, information and continuing education (in some cases of re-education) is possible the transformation of mentality and awareness of the importance of LAS and animal welfare maintenance in laboratories, since it is not only related to the quality of life of the animal inside the animal facilities (which by itself would be the most important point), but also in the issue of acquisition, reproducibility and confiability, and consequently in the ethical use by reducing the number of animals used in the research. We are convinced that this importante research problem will only be resolved when the animal welfare in the laboratory is strongly considered and investigated, and appropriate methodologies are applied in accordance with what is described in the Laws. Thus, we transform the point of view over animal model as a Bioreactor for a Biomodel, being able to associate clinical/behavioral methods, consequently, to increase the welfare animal, reproducibility and confiability of the results in preclinical trials.Barker K, Lamb SE, Toye F, Jackson S, Barrington S (2004) Association between radiographic joint space narrowing, function, pain and muscle power in severe osteoarthritis of the knee. Clinical Rehabilitation 18(7): 793-800. 